Martin HARFMANN

\title{
ÜBERSETZUNGSÜBUNGEN IM DAF-UNTERRICHT ZUR VERMITTLUNG LANDESKUNDLICHER KENNTNISSE
}

\section{Einleitung}

Im diesem Beitrag soll verdeutlicht werden, wie Übersetzungsübungen im DaF-Unterricht eingesetzt werden können, um landeskundliche Kenntnisse zu vermitteln. Zunächst wird auf die Diskussion über die Befürwortung und die Ablehnung des Einsatzes von Übersetzungsübungen im Fremdsprachenunterricht eingegangen. Weiter wird die Problematik der Übersetzung kulturgebundener Begriffe diskutiert, die auf der Grundlage eines touristischen Informationstextes zum Kölner Dom und seiner arabischen Übersetzung veranschaulicht wird. Dann wird der Begriff Übersetzung aus linguistischer Perspektive definiert und auf den Übersetzungsprozess Bezug genommen, wobei zwischen offener und verdeckter Übersetzung unterschieden wird. Im Anschluss daran werde ich eine in meinem arabischdeutschen Übersetzungsunterricht gestellte Übersetzungsaufgabe sowie die im Übersetzungsunterricht behandelte Textsorte „Lexikonartikel“ erörtern. Schließlich gehe ich auf der Basis der im Unterricht verwendeten landeskundlichen arabischen Internettexte auf Probleme ein, die sich bei der Übersetzung ins Deutsche ergeben und die im DaF- Unterricht diskutiert werden könnten, um kulturkontrastive Kenntnisse zu vermitteln. Abschließend präsentiere ich einige Schlussfolgerungen meiner Studie.

\section{Die Rolle der Übersetzung im Fremdsprachenunterricht}

Im 20. Jahrhundert wurde die Übersetzung in fremdsprachlichen Vermittlungskonzepten weitgehend ignoriert (Cook 1998, S. 117f.). In neueren Ansätzen zur Fremdsprachenvermittlung wird der Übersetzung allerdings wieder eine größere Rolle beigemessen (ebd.). Die Ablehnung von Übersetzungsübungen steht in engem Zusammenhang mit der Kritik an der Grammatikübersetzungsmethode, deren Vertreter annahmen, dass man eine 
Fremdsprache lernt, in dem man die Wörter dieser Sprache lernt und ihre grammatischen Regeln kognitiv durchdringt (Edmondson/House, S. 114.) ${ }^{1}$. Lernfortschritte werden hierbei durch grammatische Übungen und Hin- und Herübersetzungen überprüft. Aus der Kritik an der Grammatikübersetzungsmethode wurde von Vertretern der Reformbewegung die Direkte Methode entwickelt (Edmondson/House, 2006, S. 115). Bei der Direkten Methode lag der Fokus auf der Entwicklung der Sprechfähigkeiten (ebd.). Im Unterricht wurden ausschließlich zielsprachige Übungsformen präsentiert, da man davon ausging, dass ein starker Bezug zur L1 sich hemmend auf den Fremdsprachenerwerb auswirke und die Interferenz durch L1 befördere (Cook 1998, S. 117).

Die Diskussion über den Einsatz von Übersetzungsübungen im Fremdsprachenunterricht ist außerdem eng mit der Debatte darüber verknüpft, ob und in welchem Maße die Muttersprache den Erwerb einer Fremdsprache unterstützen kann (Königs 2010, S. 1042). Butzkamm (2000) spricht sich für einen durch die Lehrperson geplanten Einsatz der L1 im Fremdsprachenunterricht aus. Die Übersetzung von L2-Äußerungen in die Muttersprache eines Lernenden ist Butzkamm (2000) zufolge ein probates Mittel zur Semantisierung von neu eingeführten Äußerungen. Die Rückübersetzung könne dabei in zwei Schritten erfolgen, um ein präzises Verständnis des neu eingeführten Sprachmaterials sicher zu stellen: Zunächst wird eine idiomatische Übersetzung zur Bedeutungsklärung durch die Lehrperson vorgenommen; in einem zweiten Schritt könne eine wörtliche Übersetzung dazu dienen, die Struktur der betreffenden Äußerung zu verdeutlichen ${ }^{2}$.

Königs (2010, S. 1043) führt an, dass dem Bewusstsein von fremdsprachlichen Strukturen und Lernvorgängen in der L2 eine wichtige Rolle für die erfolgreiche Fremdsprachenaneignung beigemessen wird. Dieses Bewusstsein kann seiner Ansicht nach durch Übersetzungsübungen geschärft und beispielsweise negativer Transfer durch die Bewusstmachung von Unterschieden zwischen L1 und L2 vermieden werden (Königs 2000, S. 8).

Siehe auch Königs (2010, S. 1041f.).

$\mathrm{Zu}$ Butzkamms Argumentation für den reflektierten Einsatz der L1 sowie der Rückübersetzung im Fremdsprachenunterricht siehe auch Königs (2010, 1042ff). 
Cook (1998, S. 119) verweist darauf, dass Lerner durch den Originaltext im Rahmen einer Übersetzungsübung dazu gezwungen seien, sich auch mit solchen Bereichen des L2-Systems auseinanderzusetzen, die ihnen Schwierigkeiten bereiten und die sie bei der Bewältigung einer weniger gesteuerten Aufgabe durch eine Vermeidungsstrategie umgehen könnten.

Der vorliegenden Studie liegt die Annahme zugrunde, dass Übersetzungsübungen im Sprachunterricht eingesetzt werden können, um über Unterschiede und Gemeinsamkeiten zwischen Sprachen zu reflektieren. House (2010, S. 330) hebt hervor, dass das Übersetzen es ermöglicht, ,über Sprachspezifik und Sprachuniversalität, über Höflichkeit und Indirektheit in verschiedenen Sprachen und über die Problematik der Diskrepanz zwischen Form und Funktion beim Überwechseln von einem sprachlichen Kode in einen anderen" nachzudenken. Weiterhin macht House deutlich, dass die vergleichende Analyse von Original und Übersetzung dazu dienen kann, „versteckte Ideologien“ herauszuarbeiten (ebd.). Übersetzung kann im Sprachenunterricht dazu verwendet werden, landeskundliche und kulturkontrastive Kenntnisse zu vermitteln (ebd.). Das Übersetzen ist dabei allerdings als kommunikative Handlungen zu sehen, d.h., dass Übersetzungsaufgaben in realistische, nachvollziehbare Situationen eingebettet werden sollten, um Kommunikationsbedürfnisse der Lerner zu simulieren (House 2010, S. 328f.).

\section{Die Problematik der Übersetzung kulturgebundener Begriffe}

Im Folgenden wird eine touristische Informationsbroschüre zum Kölner Dom und seine arabische Übersetzung herangezogen, um anhand einer Übersetzungsanalyse die Problematik der Übersetzung kulturspezifischer Begriffe zu veranschaulichen. Es wird angenommen, dass eine Diskussion über Schwierigkeiten bei der Übersetzung kulturgebundener Wörter hilfreich für die Vermittlung landeskundlicher Kenntnisse im DaF-Unterricht sein kann.

Aus einer touristischen Besichtigungsbroschüre habe ich einen Abschnitt ausgewählt, der thematisch als auch die Textfunktion betreffend Übereinstimmungen aufweist mit den für die Übersetzungsaufgaben herangezogenen Texten, die für den Kurs arabisch-deutsche Übersetzung im 4. Jahr Islamwissenschaft verwendet wurden (zur Beschreibung der Textsorten 
„Besichtigungstexte“ (als Subtextsorte zu Reiseführern) und „Lexikonartikel“ (bzw. Wikipedia-Texte) siehe Abschnitt 6.). Der Abschnitt handelt von der Entstehungsgeschichte eines Bauwerks, nämlich des Kölner Doms, das von besonderer Bedeutung für den christlichen Kulturbereich ist. Ziel der Übersetzungsanalyse ist es herauszufinden, ob, und wenn ja, welche Probleme bei der deutsch-arabischen Übersetzung kulturgebundener Begriffe auftauchen können $^{3}$. Eine Diskussion über Probleme bei der Übersetzung kulturgebundener Wörter kann meiner Ansicht dazu dienen, über kulturelle Gemeinsamkeiten und Unterschiede zwischen den betreffenden Sprachkulturen zu reflektieren. Insbesondere können die betreffenden Texte dazu benutzt werden, um über möglicherweise unterschiedliche religiöse Vorstellungen nachzudenken, die sprachlich im Ausgangs- bzw. im Zieltext ausgedrückt werden.

So hat ein Vergleich zwischen deutschem Original sowie arabischem Übersetzungstext ergeben, dass christlich-konnotierte Begriffe im Ausgangstext durch nicht-religiös konnotierte oder in einem Fall durch einen islamisch konnotierten Begriff im arabischen Zieltext ersetzt wurden. Zunächst soll nun die Übersetzung der Ausdrücke im Ausgangstext diskutiert werden, die einen Bezug zum Themenbereich „Heiligenverehrung“ im christlichen Kontext aufweisen.

Im Ausgangstext wird beispielsweise angeführt, dass die Gebeine der Heiligen Drei Könige im 12. Jahrhundert in den Kölner Dom überführt wurden und ein Reliquienschrein zur Aufbewahrung der sterblichen Überreste hergestellt wurde. Im arabischen Zieltext werden für die kursiv gedruckten Begriffe Wörter benutzt, die zwar den semantischen Gehalt der Begriffe im Ausgangstext enthalten, jedoch nicht die christlich-religiösen Bedeutungskomponenten erfassen. So wird der veraltete Begriff Gebeine, der bevorzugt im deutschsprachigen religiösen Diskurs zum Ausdruck der Bedeutung „sterbliche Überreste“ verwendet wird, mit dem Wort qz̧ām (auf Deutsch: „Knochen“) übersetzt. Im religiösen arabischen Diskurs wäre statt des Wortes 'ị̄ām der Begriff rufāt angemessen, der auf sterbliche Überreste

3 Zur Problematik der Übersetzung kulturgebundener Begriffe siehe Koller (2002, S. 115-130) und Simonnaes (2002, S. 283-298). 
verweist und im religiösen Kontext verwendet werden kann ${ }^{4}$. Der Begriff

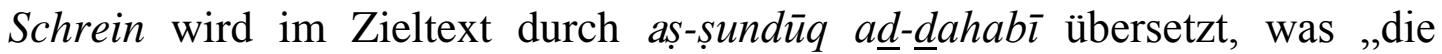
goldene Truhe" bedeutet und wiederum nicht religiös konnotiert ist. Stattdessen hätte hier der religiös konnotierte Begriff maqām als Äquivalent für Schrein benutzt werden sollen'. Schließlich wird der Begriff „Reliquien“

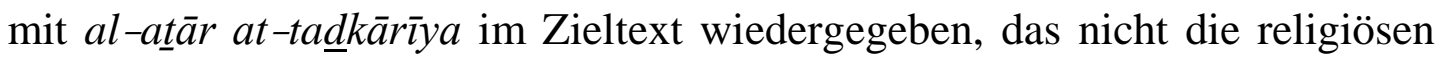
Konnotationen des ausgangssprachlichen Ausdrucks enthält und stattdessen auf „Erinnerungsgegenstände“ verweist. Um die religiösen Konnotationen zu erhalten, wäre an dieser Stelle der Begriff $\underline{d} a h \bar{a}$ ir passender gewesen ${ }^{6}$.

Abgesehen von Wörtern, die sich auf den Themenkomplex „Heiligenverehrung“ beziehen, wird im Original eine doppelchörige Kirchenanlage erwähnt, wobei das Wort Chor hier auf den Raum in der Kirche, zu dem nur Geistliche Zutritt haben und der das Kirchenschiff abschließt, verweist ${ }^{7}$. Im Zieltext wird nun der Begriff „kanīsa d̄i miḥrāb muzdawi ğ“ verwendet, das ins Deutsche rückübersetzt „Kirche mit doppelter Gebetsnische" bedeutet. Das Wort mihrāb verweist auf die Nische in einer Moschee, die die Gebetsrichtung anzeigt und ist daher islamisch konnotiert. Um den ausgangssprachlichen Ausdruck „Chor“ im Arabischen angemessen wiederzugeben, hätte der Ausdruck „kanīsa dī haikal muzdawiğ benutzt werden sollen. Der Begriff „haikal“ kann „Altar“ und auch „Tempel“ bedeuten und erfasst somit die christlich-religiösen Bedeutungskomponenten des Wortes „Chor"s.

Es kann vermutet werden, dass der Übersetzer ebenso wie ein Großteil der arabischen zielsprachigen Leser nicht mit den arabischen Begriffen vertraut sind, die allgemein zum christlich religiösen Diskurs oder im

\footnotetext{
4 Siehe Hans Wehr: Arabisches Wörterbuch für die Schriftsprache der Gegenwart und Supplement. Arabisch-Deutsch. Herausg. von Lorenz Kropfitsch, 5. Auflage Wiesbaden, 1985: Harrassowitz Verlag.

Siehe Wehr (1985).

(Ebd.).

Im „Wahrig. Deutsches Wörterbuch (2006)“ wird die Bedeutung von „Chor“ u.a. wie folgt wiedergegeben: ,den Geistlichen vorbehaltener, das Kirchenschiff abschließender Raum mit Hochaltar u. Chorgestühl“". Siehe Wehr (1985).
} 
Speziellen zum Bereich „Heiligenverehrung“ gehören. Ebenso ist denkbar, dass der Übersetzer christlich-religiöse Begriffe, die auf Heiligenverehrung verweisen, bewusst vermieden hat, um einen für die Mehrheit der arabischen Muslime verständlichen und diese nicht irritierenden Zieltext zu verfassen: Im sunnitischen Islam, dem die überwiegende Mehrheit der arabischsprachigen Menschen angehören, wird „Heiligenverehrung“ abgelehnt. Diese Form des religiösen Brauchtums wird hingegen nur von Arabern praktiziert, die der Schi'a oder der orthodoxen oder katholischen Kirche und damit Minderheiten in den arabischen Ländern zuzurechnen sind. Es scheint sinnvoll zu sein, bei der Anfertigung des arabischen Zieltextes nicht nur die christlichen Konnotationen $\mathrm{zu}$ beachten, sondern den kulturellen Kontext erläuternde Anmerkungen zum Themenbereich „Heiligenverehrung“ anzufügen.

\section{Was bedeutet Übersetzung aus linguistischer Perspektive?}

Anhand der folgenden Ausführungen soll nun geklärt werden, wie Übersetzen aus linguistischer Perspektive definiert werden kann. Die Definition soll dazu dienen, die zuvor geschilderten Probleme zur Übersetzung kulturgebundener Begriff genauer zu beschreiben.

In der vorliegenden Studie wird vorausgesetzt, dass das Konzept der Äquivalenz für die Übersetzungswissenschaft erforderlich ist. So wird davon ausgegangen, dass bei der Übersetzung ein Zieltext produziert wird, der ein funktionales Äquivalent zum Ausgangstext darstellt (House 2001, S. 260). Unter Funktion ist hierbei die Verwendung eines Textes in einem gegebenen situativen Kontext zu verstehen (House 2002, S. 103).

Der Begriff „Äquivalenz“ dient einerseits dazu, Übersetzung von anderen Formen der Textverarbeitung $\mathrm{zu}$ unterscheiden und so den Forschungsgegenstand der Übersetzungswissenschaft einzugrenzen (Koller 2004, S. 82ff.). So unterscheidet Koller (2004, S. 82) zwischen intralingualen und interlingualen Textverarbeitungsverfahren. $\mathrm{Zu}$ den intralingualen Textverarbeitungsverfahren gehören z.B. das Kommentieren oder das Erklären eines Textes (ebd.). Bei der interlingualen Übersetzung wird auf der Basis eines bereits existierenden Textes ein neuer Text in einer anderen Sprache produziert. Der Zieltext muss hierbei bestimmten Äquivalenzmaßstäben entsprechen (Koller 2004, S. 80). 
Koller (2004, S. 216) ${ }^{9}$ unterscheidet fünf Referenzrahmen, die zur genauen Beschreibungen der Äquivalenzbeziehung sowie der Klassifizierung verschiedener Äquivalenztypen verwendet werden können: Die denotative Äquivalenz, die konnotative Äquivalenz, die textnormative Äquivalenz, die pragmatische Äquivalenz und die formal-ästhetische Äquivalenz. Die von Koller (2004) vorgeschlagenen Referenzrahmen machen klar, dass Äquivalenz keinesfalls mit Identität gleichzusetzen ist, da nicht allen Äquivalenzanforderungen gleichzeitig beim Übersetzen entsprochen werden kann. Der Übersetzer ist gezwungen, eine Hierarchie von Äquivalenzanforderungen zu erstellen und eine Übersetzung zu produzieren, die diesen Prioritäten entspricht (House 1997, S. 28).

Das Konzept „Äquivalenz“ basiert auf dem Erhalten bestimmter Bedeutungsaspekte beim Prozess der Reproduktion eines Textes in einer anderen Sprache, wobei drei Aspekte von besonderer Wichtigkeit sind: 1) der semantische Aspekt von Bedeutung, 2) der pragmatische Aspekt von Bedeutung und 3) der textuelle Aspekt von Bedeutung (House 1997, S. 30ff. $)^{10}$. Diese drei Bedeutungskomponenten sollen im Folgenden kurz erläutert werden:

1) Unter dem semantischen Aspekt der Bedeutung ist die Referenzbeziehung zu verstehen, d.h., dass sprachliche Symbole auf Referenten in einer außersprachlichen Welt verweisen (House 1997, S. 30). Hierbei wird berücksichtigt, dass sich sprachliche Einheiten nicht nur auf Referenten in der real existierenden Welt beziehen können. Sprachliche Symbole können vielmehr auch auf Referenten verweisen, die die menschliche Fantasie hervorgebracht hat und die nicht in der realen Welt existieren (ebd.).

2) In der Pragmatik geht man der Beziehung zwischen den Kommunikationsteilnehmern und den von ihnen verwendeten sprachlichen Zeichen in einer bestimmten Kommunikationssituation nach. Im Mittelpunkt des Interesses steht die Frage, mit welcher kommunikativen Absicht sprachliche Symbole benutzt werden und

\footnotetext{
$9 \quad$ Siehe auch Koller (1995, S. 197f.) und Harfmann (2010, S. 37f.).

10 Siehe hierzu auch Harfmann (2010, S. 40ff.).
} 
welche Wirkung diese beim Rezipienten entfalten. In der Pragmatik geht man davon aus, dass die Analyse der kommunikativen Verwendung von Sätzen bei der Verrichtung sozialer Handlungen Informationen über die pragmatische Bedeutung von Äußerungen hervorbringen kann (ebd.). Die pragmatische Bedeutung erfasst ebenso die konnotative Bedeutung sprachlicher Symbole, geht also über den denotativen Gehalt hinaus und beinhaltet auch ihren kommunikativen Wert (ebd.).

3) Die textuelle Komponente von Bedeutung verweist auf die Herstellung unterschiedlicher Arten kotextueller Beziehungen, wie z.B. die ThemaRhema-Abfolge, pronominale Verkettung, Verwendung von Substitutionen, Koreferenzen, Ellipsen und anaphorischen Referenzen, durch die der Prozess der Textproduktion gekennzeichnet ist (House 1997, S. 31). Der Begriff „Text“ wird als Verbindung von Sätzen zu einer kohäsiven Einheit oberhalb der Satzebene definiert (ebd.).

Das Übersetzen wird in dieser Studie House (1997) folgend als das Ersetzen eines Textes in der Ausgangssprache durch einen semantisch, pragmatisch und textuell äquivalenten Text in der Zielsprache definiert ${ }^{11}$.

Ausgehend von dieser Definition können die oben geschilderten Übersetzungsprobleme der pragmatischen Ebene zugeordnet werden. Die oben angeführten christlich konnotierten Begriffe „Chor“ (bzw. „doppelchörig“), "Gebeine“ (bzw. „Gebeine der Heiligen Drei Könige“), „Reliquien“, „Schrein“ (bzw. „Reliquienschrein“) verdeutlichen die Absicht des Autors, im Rahmen der Erläuterung der Entstehungsgeschichte des Kölner Doms, die besondere Bedeutung zu betonen, die dem Kölner Dom aus christlichreligiöser Perspektive zukommt. Die erwähnten Begriffe verweisen auf die Merkmale des Kölner Doms, die diesen aus christlich-religiöser Sicht einzigartig machen. Hierzu gehört insbesondere, dass sich im Kölner Dom die sterblichen Überreste der Heiligen Drei Könige befinden sollen. Die verwendeten Begriffe „Gebeine“, „Reliquien“ und „Schrein“ unterstreichen die Wichtigkeit der Heiligenverehrung im Katholizismus. Dieser Aspekt, der

$11 \quad$ Siehe auch Harfmann (2010, S. 42). 
im Original hervorgehoben wird, wird in der Übersetzung außer Acht gelassen, denn die sich auf Heiligenverehrung beziehenden Begriffe im Ausgangstext werden im Zieltext durch nicht-religiös konnotierte Wörter ersetzt.

Der Begriff „Chor“ (bzw. „,doppelchörig"), der sich auf einen bestimmten Bereich in einer christlichen Kirche bezieht und daher christlich konnotiert ist, wird zudem durch einen Begriff in der Übersetzung wiedergegeben, der sich auf einen bestimmten Bereich einer Moschee bezieht, nämlich mihrāa (Gebetsnische in einer Moschee) (bzw.,,kanīsa d̄i miḥrāb muzdawi $\check{g}^{\prime \prime}=$ Kirche mit doppelter Gebetsnische). Auch hierdurch wird die spezifisch christliche Komponente des Originaltextes im Übersetzungstext nur abgeschwächt wiedergegeben. Insofern ist der Zieltext auf pragmatischer Ebene nicht äquivalent zum Ausgangstext.

\section{5. $\quad$ Offene und verdeckte Übersetzung}

Die von House (1997) vorgeschlagene Übersetzungstypologie wird im Folgenden erläutert, der zufolge zwischen offener und verdeckter Übersetzung zu unterscheiden ist ${ }^{12}$. Der Ausdruck ,offene Übersetzung“ bezieht sich auf einen Zieltext, der vom Rezipienten als Übersetzung erkannt werden kann, da kulturspezifische Kennzeichen des Ausgangstextes im Zieltext erhalten bleiben. Es wird kein funktionales Äquivalent zum Ausgangstext produziert, um den Adressaten anzusprechen. Eine offene Übersetzung anzufertigen ist dann sinnvoll, wenn das Original in besonderer Weise an die Ausgangskultur gebunden ist. Die Übersetzung ermöglicht es einem Rezipienten, der die Ausgangssprache nicht oder nicht in ausreichendem Maße beherrscht, Zugang zum Original und zur Textfunktion zu erhalten, die der Text im ausgangskulturellen Rahmen hat oder gehabt hat.

Eine verdeckte Übersetzung zeichnet sich dagegen dadurch aus, dass sie nicht als Übersetzung zu erkennen ist. Der Zieltext wird mit dem Ziel angefertigt, wie ein Original rezipiert zu werden. Die Übersetzung kann als ein funktionales Äquivalent zum Original betrachtet werden. Kulturspezifische Merkmale des Ausgangstextes werden mit Hilfe eines kulturellen Filters

$12 \mathrm{Zu}$ offenen Übersetzung siehe auch Harfmann (2010, S. 118f.). 
erkannt und „heraus gefiltert", d.h., nicht in den Zieltext übernommen. Stattdessen wird ein Zieltext produziert, der den Erwartungsnormen der Zielkultur entspricht ${ }^{13}$. Eine verdeckte Übersetzung sollte dann angefertigt werden, wenn der Ausgangstext nicht in besonderem Maße an die Ausgangskultur gebunden ist und das Original und die Übersetzung potentiell gleich relevant für die Ausgangs- und die Zielkultur sind.

Die von House (1997) vorgeschlagene Übersetzungstypologie wird für die folgende Studie übernommen, wobei einschränkend hinzuzufügen ist, dass auch verdeckt übersetzte Text sich in einigen Bereichen durch Abweichungen von zielsprachigen Erwartungsnormen auszeichnen bzw. Übereinstimmungen mit ausgangssprachlichen Textkonventionen aufweisen können. So hat ein Vergleich von deutschen Werbetexten mit ihren arabischen Zieltexten ergeben, dass die Übersetzungen zwar ein hohes Maß an sprachlicher Präzision aufweisen und auf diese Weise an arabische Textkonventionen angepasst wurden ${ }^{14}$. Andererseits fehlen in diesen Zieltexten aber einige für arabische Werbetexte typischen Stilmittel ${ }^{15}$.

Bezüglich der touristischen Broschüre zum Kölner Dom wird davon ausgegangen, dass sie verdeckt übersetzt werden sollte, da der Text für Besucher der Sehenswürdigkeit, die der arabischen Zielkultur zugehörig sind, potentiell genauso relevant ist, wie für Besucher des Gebäudes, die Mitglieder der Ausgangskultur sind. Dementsprechend sollte die Übersetzung der Broschüre funktional äquivalent zum Original sein. Es wird angenommen, dass für arabischsprachige Besucher des Kölner Doms insbesondere von Interesse ist, Informationen darüber zu erhalten, welche Bedeutung dem Bau aus Perspektive der Ausgangskultur zukommt. Auf diese Weise kann durch

\footnotetext{
13 House (2005, S. 84). Siehe auch Harfmann (2010, S. 119f.).

14 Siehe Harfmann (2010). Stock (2005, S. 38) zufolge gehört ein präzise Ausdrucksweise, bei der es um das Erfassen von Nuancen geht, zur arabischen Sprachkultur. Arabische Werbetexte sind ihr zufolge durch Elemente einer präzisen Ausdrucksweise geprägt (Stock 2005, S. 116ff).

15 Bei den untersuchten arabischen Zieltexten konnte beispielsweise nicht das Stilmittel sağ (Reimprosa) nachgewiesen werden, das Stock (2005, S. 118) zufolge ein typisches Element arabischer Werbetexte darstellt (Harfmann 2010, S. 232).
} 
den Besuch des Kölner Doms etwas über den kulturellen Kontext gelernt werden, in dem die Kirche entstanden ist.

6. Beschreibung der im arabisch-deutschen Übersetzungsunterricht
verwendeten Textsorte und der Übersetzungsaufgabe

Für das Seminar zur arabisch-deutschen Übersetzung im 4. Studienjahr des Faches „Islamwissenschaft auf Deutsch“ der Azhar Universität wurden Teile landeskundlicher Sachtexte in arabischer Sprache aus dem Internet ausgewählt, die sich thematisch mit dem historischen Hintergrund bedeutender islamischer Bauwerke bzw. Institutionen auseinandersetzen und die ins Deutsche übersetzt werden sollten. Die Texte handelten von der AzharMoschee, der Azhar Universität sowie der Șalāḥ ad-Dīn-Zitadelle und waren der Internetseite Wikipedia entnommen ${ }^{16}$.

Lehrziel des von mir durchgeführten Übersetzungsunterrichts ist neben der Verbesserung der Übersetzungskompetenz auch der Ausbau der anderen vier sprachlichen Fertigkeiten Hören, Lesen, Schreiben und Sprechen. Hierbei stütze ich mich auf Königs (2000), der u.a. davon ausgeht, „dass “Übersetzen“ nicht isoliert von anderen Fertigkeiten geschult, sondern im Gegenteil in diese Schulung integriert werden soll“'17 (S. 11). So kann die Sprachverarbeitung als ein Versuch gedeutet werden, neues Sprachwissen $\mathrm{zu}$ bereits bestehenden Sprachwissensstrukturen in Bezug zu setzen (Königs 2000, S. 9). Übersetzungsübungen dienen dann dem Zweck, bereits vorhandenes Sprachwissen zu aktivieren und für die Lösung der Übersetzungsaufgabe einzusetzen (Königs 2000, S. 11).

\footnotetext{
16 Siehe unter (die Azhar-Moschee) الجامع الأزهر http://ar.wikipedia.org/wiki/\%D8\%A7\%D9\%84\%D8\%AC\%D8\%A7\%D9\%85\%D8\%B9_\% D8\%A7\%D9\%84\%D8\%A3\%D8\%B2\%D9\%87\%D8\%B1\#.D8.A7.D9.84.D8.A3.D8.B2.D9.8 7.D8.B1_.D9.88.D8.AB.D9.88.D8.B1.D8.A9 .D9.8A.D9.88.D9.84.D9.8A.D9.88 (Zugriff am 13.08. 2011), und unter جامعة الأزهر (die Azhar Universität) http://ar.wikipedia.org/wiki/\%D8\%AC\%D8\%A7\%D9\%85\%D8\%B9\%D8\%A9 \%D8\%A7\% D9\%84\%D8\%A3\%D8\%B2\%D9\%87\%D8\%B1 (Zugriff am 13.08. 2011), قلعة صلاح الدين Sowie unter (die Șalāh ad-Dīn-Zitadelle) http://ar.wikipedia.org/wiki/\%D9\%82\%D9\%84\%D8\%B9\%D8\%A9 \%D8\%A7\%D9\%84\%D 9\%82\%D8\%A7\%D9\%87\%D8\%B1\%D8\%A9 (Zugriff am 14.08. 2011). 
Die Übersetzungsaufgabe wurde in eine realistische Kommunikationssituation eingebettet, indem die Studierenden dazu aufgefordert wurden, die Internetexte für Studierende der Partneruniversität Vechta zu übersetzen. Als Zweck der Übersetzungsübung wurde angegeben, Zieltexte zu produzieren, die es Studierenden der deutschen Partneruni ermöglichen, Kenntnisse über den kulturellen Hintergrund der Studierenden und Mitglieder der Azhar Universität zu erwerben, um sich auf diese Weise auf einen Studienaufenthalt an dieser Universität vorzubereiten.

Weiterhin wurde vorgegeben, den Text verdeckt zu übersetzen, also einen weitgehend den Erwartungsnormen der Zieltextadressaten gerechten Zieltext zu produzieren.

Die Textsorte Lexikonartikel im Deutschen wurden diskutiert und auf die Kommunikationssituation und die Textfunktionen von Lexikonartikeln sowie auf ihre sprachlichen Merkmale eingegangen, um den Studierenden Orientierungspunkte für die Produktion des Zieltextes an die Hand zu geben. Insbesondere wurde die vom Prototyp des Lexikonartikels etwas divergierende Variante „Wikipedia-Text“ berücksichtigt, wobei die Diskussion auf der Textsortenanalyse von Fandrych/Thurmair (2011) aufbaute.

Fandrych/Thurmair (2011) zufolge sind Lexikonartikel vor allem durch eine konstatierend-assertierende, wissensbereitstellende Textfunktion gekennzeichnet $^{18}$. Solch eine Funktion erfüllen sach- bzw. fachbezogene Texte, in denen das präsentierte Wissen als allgemein gültig bzw. objektiv zutreffend dargestellt wird. Eine argumentative Untermauerung der dargebotenen Informationen erübrigt sich, da vorausgesetzt wird, dass die Rezipienten diese nicht infrage stellen sondern akzeptieren.

Lexikonartikel werden mit dem Ziel produziert, Wissenslücken potentieller Leser zu schließen. Im Gegensatz zu Wörterbuchartikeln, die ebenfalls der Textsorte „Lexikonartikel“ zuzurechnen sind, werden in enzyklopädischen Artikeln vor allem substantivische Lemmata, darunter Eigennamen von Ländern, Städten und Persönlichkeiten des öffentlichen Lebens, erläutert, wobei in erster Linie „der vom Lemma bezeichnete Realitätsausschnitt im Vordergrund des Eintrags, häufig in seiner fachlichen,

18 Siehe Fandrych/Thurmair (2011, S. 30) zur Beschreibung der konstatierend-assertierenden, wissensbereitstellenden Funktion. 
historischen, gesellschaftlichen oder lebensweltlichen Funktion, Bedeutung und Systematik“steht (Fandrych/Thurmair 2011, S. 90).

Da es sich bei Lexikonartikel um wissensbezogene Texte handelt und dem Autor normalerweise nicht in unbegrenztem Maße Raum zur Verfügung steht, müssen Lexikonartikel in relativ hohem Maße sprachlich verdichtet $\operatorname{sein}^{19}$. Daher sind in Lexikonartikeln Formen des Nominalstils sowie einer passivischen Ausdrucksweise vorherrschend. Auch werden in Lexikonartikeln relativ häufig Parenthesen sowie elliptische Äußerungen verwendet, wobei insbesondere die Weglassung der Kopula häufig $\mathrm{zu}$ beobachten ist. Lexikonartikel enthalten darüber hinaus wenige Konnektoren und Pronomina, da der Textproduktion die Zielsetzung zugrunde liegt, nur die wichtigsten Informationen zum behandelten Themengegenstand in Form von präzisen Formulierungen darzulegen.

Zwar zeichnen sich Wikipedia-Texte durch zahlreiche Merkmale aus, die prototypische Enzyklopädien aufweisen. Der Inhalt der Wikipedia-Texte sowie ihre Aufnahme in das Internetlexikon können jedoch kaum durch eine Redaktion oder durch Herausgeber kontrolliert werden (Fandrych/Thurmair 2011, S. 106). Verfasser von Wikipedia-Texten unterliegen nicht in dem Maße wie Autoren nicht-elektronischer Enzyklopädie-Artikeln Beschränkungen bezüglich der Artikellänge. Daher ist zu erwarten, dass beim Verfassen dieser Texte weniger stark auf den Nominalstil sowie auf andere Mittel der sprachlichen Verdichtung zurückgegriffen wird. Elektronische Texte weisen zudem häufig viele Verknüpfungen zu anderen Wikipedia-Texten oder zu Webseiten außerhalb der Wikipedia-Enzyklopädie auf.

Es soll darauf hingewiesen werden, dass die für den Übersetzungsunterricht herangezogenen Texte Übereinstimmungen hinsichtlich der Textfunktion mit der von Fandrych/Thurmair (2011) diskutierten Subtextsorte „Besichtigungstexte“ aufweisen, die zu den Großtexten „Reiseführer“ gehören (Fandrych/Thurmair 2011, S. 52ff.) ${ }^{20}$. So gilt für Besichtigungstexte, dass sie sich häufig durch eine „Verbindung

19 Siehe Fandrych/Thurmair (2011, S. 108ff.) zur Beschreibung der sprachlichen Merkmale von Lexikonartikeln.

20 Als weitere Subtexte zu Reiseführern nennen Fandrych/Thurmair (2011, S. 53f.) Orientierungstexte, Hintergrundtexte und Ratgebertexte. 
historisch-narrativer und am Objekt orientierter beschreibender Perspektiven“ auszeichnen (Fandrych/Thurmair 2011, S. 62). Die erzählende Vorgehensweise in Besichtigungstexten zeigt sich u.a. an der Verwendung des Präteritums, das Beschreiben kann anhand der Benutzung des Präsens verdeutlicht werden, so dass in Besichtigungstexten beide Tempora zu beobachten $\operatorname{sind}^{21}$. Der Wechsel zwischen historisch-narrativem und beschreibendem Vorgehen lässt sich ebenso in den von mir diskutierten Wikipedia-Texten wie auch anhand der Touristenbroschüre mit Informationen über den Kölner Dom beobachten. Dass es sich bei der Touristenbroschüre um einen Besichtigungstext handelt, wird auch dadurch klar, dass diese für Besucher im Kölner Dom ausgelegt war $^{22}$. Ein erzählendes Vorgehen wird ebenso an der chronologischen Nennung von Jahreszahlen deutlich, durch die sich alle verwendeten Texte auszeichnen. Im Unterschied zu Besichtigungstexten in Reiseführern, sind aber die in dieser Studie verwendeten Texte nicht kommerziell, der Zugang zu ihnen ist ohne Bezahlung möglich.

\section{Die Diskussion von Übersetzungsproblemen im Unterricht}

Im Folgenden sollen Übersetzungsprobleme erläutert werden, die im Unterricht mit den Studierenden diskutiert wurden, wobei ausgehend von der unter Abschnitt 3 angegebenen Definition des Übersetzens zwischen Problemen auf der semantischen, der pragmatischen und der textuellen Ebene unterschieden wird.

\section{Probleme auf der semantischen Ebene}

In den Texten, die von der Azhar-Moschee und der Azhar Universität handeln, befinden sich Äußerungen, in denen angegeben wird, an welchem Tag im Monat und in welchem Jahr sich etwas ereignete, wobei die Jahreszahl

$21 \quad$ Weinrich (2007) unterscheidet ebenso zwischen besprechenden und erzählenden TempusRegistern, wobei er Präsens, Perfekt und Futur dem Besprechen und Präteritum und Plusquamperfekt dem Erzählen zuordnet (S. 198ff.).

22 Ich habe die Broschüre und ihre arabische Übersetzung während meines Besuches dort im Jahre 2008 erhalten. 
nach christlichem und islamischem Kalender genannt wird. Folgende Äußerung aus dem Text über die Azhar Universität soll als Beleg angeführt werden:

Beispiel 1)

Ausgangstext

وصلى فيه الخليفة المعز لدين الهه الفاطمي ... صلاة الجمعة الأولى من شهر رمضان سنة

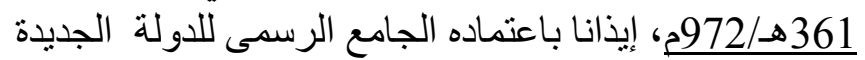

Zieltext

Der fatimidische Kalif al-Mu'izz li-Dīn Allah ... verrichtete in ihr das Freitagsgebet am 1. des Monats Ramadān im Jahr 361 nach der Hiğra bzw. 972 n. Chr., wobei er verkündete, sie als offizielle Moschee des neuen Reiches anzuerkennen.

Eine weitere zweifache Angabe von Jahreszahlen befindet sich am Beginn des Textes.

Vom zielsprachigen Leser kann angenommen werden, dass er über keine Kenntnisse der islamischen Monatsnamen verfügt und dass ihm das Vorwissen fehlt, um die islamische Datumsangabe in einen Kontext einzuordnen. Häufigere zweifache Nennung der Jahreszahl ist zudem als fragwürdig im Deutschen einzustufen, da diese die Rezeption des Textes erschwert. Die durchgängige Nennung von Jahreszahlen nach christlichem und islamischem Kalender ist selbst in deutscher islamwissenschaftlicher Literatur nicht üblich. Im Arabischen hingegen ist die doppelte Datumsangabe beispielsweise auf den Titelseiten von Zeitungen zu beobachten.

Es stellt sich die Frage, ob die wiederholte zweifache Nennung der islamischen Jahreszahlen im deutschen Zieltext sinnvoll ist, wenn, wie oben bereits festgestellt, ein verdeckte Übersetzung angestrebt wird, die vom Rezipienten nicht als Übersetzung erkannt wird. Mir erscheint eine einmalige zweifache Jahreszahlangabe mit Nennung des islamischen Datums beim erstmaligen Auftreten dieser Angabe im Text dann als sinnvoll, wenn eine erläuternde Fußnote hinzugefügt wird, in der u.a. darauf hingewiesen wird, dass sich die islamische Datierung nach der Emigration (Hiğra) des Propheten Muhammad von Mekka nach Medina richtet und an Mondjahren orientiert ist, wobei der Monatsanfang aufgrund der Sichtbarkeit des Vollmondes bestimmt 
wird. Hierdurch wäre gewährleistet, dass die anvisierten zielsprachigen Leser, nämlich Studierende der Universität Vechta, etwas Neues über den kulturellen Hintergrund der Mitglieder ihrer Partneruniversität Azhar erführen.

Probleme bei der Übersetzung, die ebenfalls auf der semantischen Ebene anzusiedeln sind, werden anhand von Begriffen in den diskutierten Texten deutlich, die sich auf Ämter bzw. Funktionen bestimmter historischer Persönlichkeiten oder auf Herrscherdynastien aus dem islamischen Kontext beziehen. So wird beispielsweise im Text über die Azhar-Moschee mithilfe des Ausdrucks „al-hulafā' al-fățimiyīna“ (die fatimidischen Kalifen) auf die Kalifen der fatimidischen Herrscherdynastie verwiesen. Bei den Begriffen "fatimidisch“ und „Kalif" handelt es sich um Lehnwörter aus dem Arabischen, deren jeweilige genaue Bedeutung dem zielsprachigen Leser unbekannt sein dürfte. Das Adjektiv „fatimidisch“ ist vom Substantiv „Fatimide“ abgeleitet. Dieses ist wiederum eine Ableitung von „Fatima“, dem Namen der Tochter des Propheten Muhammads, auf die fatimidische Herrscher sich genealogisch zurückzuführen suchten ${ }^{23}$.

Das Wort „Kalif“" ist dem arabischen „Haliffa“ entlehnt, das „Stellvertreter des Gesandten Gottes“ bedeutet und sich somit auf das Haupt der islamischen Theokratie bezieht ${ }^{24}$.

Zahlreiche Verweise auf Ämter (wie z.B. Sultan, Wezir, Khedive) und Herrscherdynastien (Aiyubiden und Mamluken) aus dem islamischen Kulturbereich finden sich im Text über die Șalāḥ ad-Dīn-Zitadelle. Meines Erachtens ist es angebracht, diese in der Islamwissenschaft zu Fachwörtern zählenden Begriffe nicht im Text sondern in einem Glossar zu erläutern, um den zielkulturellen Lesern vertiefte Kenntnisse über den islamischen Kulturraum zu vermitteln und so das Textverständnis zu sichern.

23 Zur Legitimation des ersten fatimidischen Kalifen 'Abdallah ,al-Mahdī“ durch den Verweis auf genealogische Abstammung von der Prophetentochter Fațima siehe Endress (1982, S. 56) und Halm (1994, S. 169).

24 Zur Bedeutung des Begriffs „Halīfa“ siehe Noth (1994, S. 75f.). 


\section{Probleme auf der pragmatischen Ebene}

Im Folgenden soll auf Begriffe in den diskutierten Texten aufmerksam gemacht werden, die islamisch konnotiert sind. So wird im Text über die Azhar-Moschee mit dem Ausdruck ,tamma fatḥ... 'alā yadd“ (semantisch: wurde erobert durch) auf die islamische Eroberung Ägyptens verwiesen:

Beispiel 2)

Ausgangstext

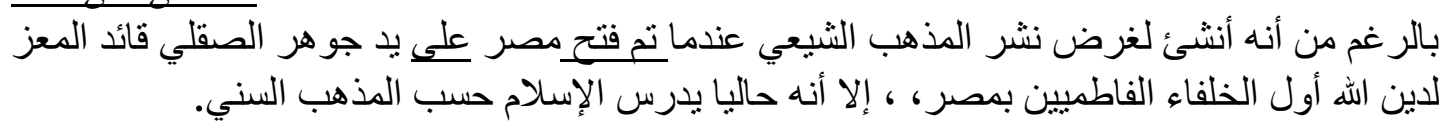
Zieltextvariante a)

Obwohl sie (die Azhar-Moschee, Anmerkung des Autors) zum Zwecke der Verbreitung der schiitischen Lehrmeinung ins Leben gerufen wurde, als Ägypten mithilfe von Ğauhar al-Saqali, dem Oberbefehlshaber unter dem Kalifen al-Mu'izz li-Dīn Allah, dem ersten fatimidischen Kalifen in Ägypten, triumphal erobert wurde, wird heute jedoch in ihr die sunnitische Lehrmeinung unterrichtet.

Im arabisch-deutschen Wörterbuch von Hans Wehr ${ }^{25}$ wird der Begriff „fatḥ" u.a. mit „Öffnung“ übersetzt. Die Pluralform futūḥ bzw. futūhāat wird mit „Eroberung“, „Sieg“, „Triumph“ und „Errungenschaften“ wiedergegeben. Beim Verb „fatah 'alā" gibt Wehr u.a. die Bedeutung ,in die Gewalt geben von Gott" an. Die im Wörterbuch von Wehr gemachten Angaben verweisen darauf, dass „fath" " positiv religiös konnotiert ist ${ }^{26}$. Den passivischen Ausdruck „tamma fatḥ 'alā yadd“ mit „,wurde erobert“ „,neutral“" zu übersetzen würde heißen, die konnotiven Bedeutungen im Zieltext außer Acht zu lassen. Um auf pragmatischer Ebene Äquivalenz zu erreichen, bietet es sich z.B. an, die Formulierung ,wurde triumphal erobert" bei der Produktion des Zieltextes zu wählen. Als problematisch erweist sich hierbei, dass die Wiedergabe der positiven Konnotationen des Ausdrucks bei den zielsprachigen Lesern Irritationen auslösen könnte. Die pragmatisch äquivalente

\footnotetext{
25 Siehe Wehr (1985).

26 Noth (1994, S. 62) gibt den Begriff futū in seiner Beschreibung der arabisch-islamischen Expansion zwischen 632 und 750 mit dem Ausdruck ,große Eroberungen“ wieder.
} 
Übersetzungsvariante würde Studierende der Universität Vechta jedoch mit einer Bewertung der islamischen Eroberung Ägyptens ,aus muslimischer Perspektive" konfrontieren und sie auf diese Weise näher an das Denken und Empfinden vieler Mitglieder ihrer Partneruniversität heranführen.

\section{Probleme auf der textuellen Ebene}

$\mathrm{Zu}$ den Problemen auf der textuellen Ebene gehören zum einen elliptische Äußerungen, die bereits bei der Rezeption des Originals zu Verständnisschwierigkeiten führen. So beginnt der Text über die AzharMoschee mit der Nennung des Namens „Azhar-Moschee“, an die Jahreszahlen in Klammern angefügt werden. Zur Verwirrung trägt bei, dass es sich um eine Angabe einer Zeitspanne nach christlichem Kalender handelt, die zwei Jahre und um eine Nennung einer Zeitspanne nach islamischem Kalender, die fünf Jahre umfassen. Für den unkundigen Leser ist es erforderlich, zu recherchieren, um zu verstehen, auf welche Zeitspannen der Autor sich beziehen könnte:

Beispiel 3)

Ausgangstext

Zieltextvariante a)

$$
\text { الجامع الأزهر (359 361 هجرية)/ (970 975 م). }
$$

Die Azhar-Mosche (359-361 nach der Hị̆ra) (970-975 n. Chr.)

Ein Blick auf den Paralleltext zur Azhar Universität bei Wikimedia in deutscher Sprache macht deutlich, dass sich die erstgenannte Zeitspanne nach islamischer Zeitrechnung auf die Bauzeit der Moschee beziehen könnte (970972 n. Chr.), während die zweitgenannte nach christlicher Zeitrechnung möglicherweise auf die Zeitspanne zwischen Baubeginn und Aufnahme der Lehrtätigkeit verweist ${ }^{27}$. Für die Erstellung des Zieltextes ist es meiner Ansicht nach wichtig, die elliptische Konstruktion im Ausgangstext in einen voll ausformulierten Satz zu übertragen, damit die zielsprachlichen Leser die

27 Text zur Azhar-Universität auf Wikepedia unter: http://de.wikipedia.org/wiki/Al-AzharUniversit\%C3\%A4t (Zugriff am 15.09. 2011). 
Äußerung verstehen können. Folgende zielsprachliche Äußerung kann als Lösungsmöglichkeit mit Studierenden diskutiert werden:

Beispiel 3)

Zieltextvariante b)

Die Azhar-Moschee wurden zwischen den Jahre 970 und 972 erbaut. Im Jahre 975, nach islamischer Zeitrechnung 365 nach der Hiğra, wurde die Lehrtätigkeit in der Moschee aufgenommen.

Da es sich um die erste Zeitangabe nach islamischem Kalender handelt, ist, wie bereits oben erwähnt, eine erläuternde Fußnote zum islamischen Mondkalender angebracht.

Im folgenden Beispiel 4) aus dem arabischen Originaltext zur AzharMoschee, das das bereits erwähnte Beispiel 2) enthält, aber um eine Äußerung erweitert ist, werden Sätze durch Pronomen miteinander verkettet. Außerdem wird an dem Beispiel eine äußerst komplexe syntaktische Struktur anhand der Verwendung von Konnektoren sichtbar, die im Falle einer offenen Übersetzung die Rezeption der zielsprachigen Äußerung erschweren würde:

Beispiel 4)

Ausgangstext

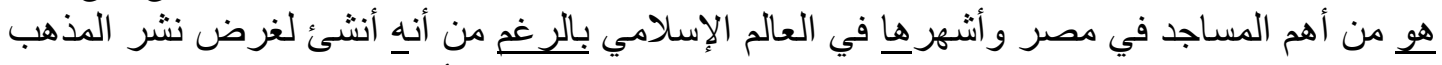

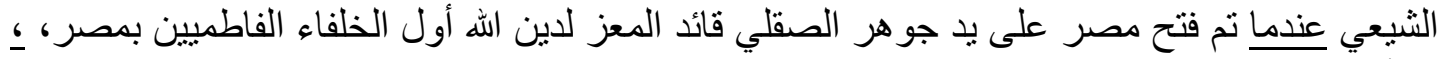

\section{Zieltextvariante a)} إلا أنه حاليا يدرس الإسلام حسب الدذهب السني

Sie gehört zu den wichtigsten Moscheen in Ägypten und zu den berühmtesten in der islamischen Welt. Obwohl sie zum Zwecke der Verbreitung der schiitischen Lehrmeinung ins Leben gerufen wurde, als Ägypten mithilfe von Ğauhar al-Saqali, dem Oberbefehlshaber unter dem Kalifen al-Mu'izz li-Dīn Allah, dem ersten fatimidischen Kalifen in Ägypten, triumphal erobert wurde, wird heute jedoch in $\underline{\mathrm{ihr}}$ die sunnitische Lehrmeinung unterrichtet.

Zusätzlich zu der an der Struktur des Originals orientierten Zieltextvariante a) soll nun im Unterricht auf Möglichkeiten der Anpassung der Struktur an stilistische Konventionen im Deutschen hingewiesen werden, 
um gemeinsam eine verdeckte Übersetzung zu erarbeiten. Wie unter Kapitel 5) bereits angeführt, zeichnet sich die Textsorte „Lexikonartikel“ im Deutschen u.a. durch verminderten Gebrauch von Pronomina und Konnektoren sowie die relativ häufige Verwendung von Parenthesen aus, um einen prägnanten sprachlichen Ausdruck zu gewährleisten. Syntaktisch weniger komplexe Zieltextvarianten sollten daher zur Diskussion gestellt werden, bei denen die Rezeption erleichtert wird, indem Schlüsselbegriffe wiederholt oder substituiert und Äußerungen nicht pronominal verkettet werden und außerdem weniger Konnektoren benutzt werden. Im Übersetzungsunterricht wurde der Paralleltext zur Azhar Universität von Wikipedia eingesetzt, um zu diskutieren, durch welche Strukturen in diesem Text Sätze miteinander verbunden werden: Folgender Beispielsatz aus dem Paralleltext verdeutlicht, wie das Textthema durch Substitution fortgeführt werden kann:

„Die Azhar-Universität (arabisch جامعة الأزهر dschami 'at al-azhar,die Blühende') in Kairo ist eine der angesehensten Bildungsinstitutionen der islamischen Welt und nach der Universität Al-Qarawiyyin von Fès - gegründet im Jahre 859 - die zweitälteste Madrasa der islamischen Welt. ${ }^{\text {'28 }}$ (Hervorhebungen von mir, M.H.).

Nach Analyse der Verknüpfungsmittel im Paralleltext, in dem der Schlüsselbegriff „Azhar-Universität“ durch andere Wörter substituiert wird, die auf den gleichen außersprachlichen Referenten verweisen und eine Parenthese realisiert wird, kann folgende Zieltextvariante vorgeschlagen werden:

Beispiel 4)

Zieltextvariante b)

Die Azhar-Moschee gehört zu den wichtigsten Moscheen in Ägypten und zu den berühmtesten in der islamischen Welt. Diese Bildungsinstitution wurde zum Zwecke der Verbreitung der schiitischen Lehrmeinung ins Leben gerufen - Ägypten wurde mithilfe von Ğauhar al-Saqali, dem Oberbefehlshaber unter dem Kalifen al-Mưizz li-Dīn Allah, dem ersten fatimidischen Kalifen in

28 Siehe Al Azhar Universität:

http://de.wikipedia.org/wiki/Al-Azhar-Universit\%C3\%A4t (Zugriff am 15. 09. 2011). 
Ägypten, triumphal erobert. Heute wird in der Azhar-Moschee jedoch die sunnitische Lehrmeinung unterrichtet.

\section{Schlussfolgerungen}

In dieser Studie habe ich versucht zu zeigen, auf welche Weise Übersetzungsübungen im DaF-Unterricht eingesetzt werden können, um landeskundliche Kenntnisse zu vermitteln. Meiner Ansicht nach ist es insbesondere sinnvoll, solche Texte übersetzen zu lassen, in denen explizit landeskundliche Informationen über die Ausgangskultur vermittelt werden sollen. DaF-Studierende werden durch solche Aufgaben dazu angeregt, sich darüber Gedanken zu machen, wie diese landeskundlichen Informationen Lesern der Zielkultur angemessen vermittelt werden können, die über andere Wissensvoraussetzungen als die Leser der Ausgangskultur verfügen.

Weiterhin bieten sich solche Texte für die Übersetzung an, die für Rezipienten der Zielkultur potentiell gleichermaßen relevant sind wie für Rezipienten der Ausgangskultur und daher verdeckt zu übersetzen sind. DaFStudierende, die verdeckt übersetzen, müssen ihr Wissen über die Ausgangsund die Zielkultur aktivieren, um einen zielkulturadäquaten Übersetzungstext produzieren zu können.

Das Wortschatzwissen, insbesondere das Wissen über kulturspezifische Wörter in den bei der Übersetzung involvierten Sprachen, ist von großer Bedeutung für das Verständnis kultureller Phänomene. Die Übersetzung kulturspezifischer Wörter stellt eine Herausforderung für Übersetzer dar, da sie darüber reflektieren müssen, wie das zu erwartende geringere Vorwissen des zielsprachlichen Lesers kompensiert werden kann, um das Verständnis des Zieltextes zu sichern.

Besonders wichtig für die Übersetzung ist das Wissen darüber, wie ein sprachlicher Ausdruck in einer bestimmten soziokulturellen Situation verwendet wird bzw. das Wissen über die pragmatische Bedeutung dieses Ausdrucks (House 2004, S. 496). Nicht nur der semantischen Gehalt sondern auch die konnotativen Bedeutungen sprachlicher Ausdrücke müssen berücksichtigt werden, um einen funktional äquivalenten Text produzieren zu können. Anhand der von mir präsentierten Übersetzungsprobleme sollte deutlich werden, dass ein auf pragmatischer Ebene äquivalenter Zieltext den 
zielsprachigen Lesern Zugang zu möglicherweise als fremd empfundenen religiösen Auffassungen ermöglichen kann. Hierdurch wird meines Erachtens ein wichtiger Zweck der Übersetzung deutlich. Grundsätzlich dient Übersetzung dazu, Mitgliedern einer Zielkultur die Rezeption eines ausgangssprachlichen Textes zu ermöglichen, die diese Ausgangssprache nicht oder nicht ausreichend beherrschen. Die Rezeption eines solchen Zieltextes kann insbesondere dann von Interesse sein, wenn dieser Text den Leser über bisher nicht erschlossene Wissensbereiche informiert und ihn mit bislang unbekannten Einstellungen oder Präferenzen konfrontiert.

Sprache wird in der vorliegenden Studie zunächst als eine soziale Erscheinung betrachtet, die eng mit Kultur verbunden ist (ebd.). Vorausgesetzt wird, dass die Bedeutung eines sprachlichen Ausdrucks nur verstanden werden kann, wenn der ihn umgebende kulturelle Kontext nicht außer Acht gelassen wird (ebd.).

Wie oben angeführt geht es beim Übersetzen um das Erhalten bestimmter Aspekte der Bedeutung beim Übertragen eines Ausgangs- in einen Zieltext. Der Begriff „Bedeutung“ ist somit zentral für die Übersetzung und der kulturelle Kontext des Ausgangs- und des Zieltextes muss beachtet werden, sowohl bei der Bewertung einer Übersetzung als auch bei der Produktion eines Zieltextes (ebd.). Am Beispiel der doppelten Angabe von Jahreszahlen - nach christlichem und islamischem Kalender - in arabischen Ausgangstexten wird deutlich, dass der Zieltextleser im Falle einer wortgetreuen Übersetzung und zweifachen Nennung der Jahreszahlen an entsprechender Stelle im Zieltext zusätzliche, den Kontext erläuternde Angaben zur Datierung des islamischen Mondkalenders erhalten sollte, um die Zeitangaben kontextuell einordnen zu können. Gleiches gilt, wenn deutsche Begriffe mit christlichen Konnotationen, die auf den Brauch der Heiligenverehrung verweisen, ins Arabische übersetzt werden, denn auch hier kann nicht vorausgesetzt werden, dass die arabischsprachigen zielkulturellen Leser über genügend Informationen verfügen, um diese Begriffe kontextuell einordnen zu können.

Abschließend weise ich darauf hin, dass in dieser Studie ein anthropologisches Konzept von Kultur vorausgesetzt wird, das sich auf die allgemeine Lebensart einer Gemeinschaft oder Gesellschaft bezieht. Dieses 
Konzept unterscheidet sich von der humanistischen Kulturauffassung, welches das „kulturelle Erbe“ einer Gemeinschaft erfasst, d.h. die exklusive Produkte einer Gesellschaft in den Bereichen Literatur, Kunst und Musik (ebd.). Bei dem anthropologischen Konzept von Kultur geht es um Orientierungsmuster, an denen Mitglieder einer Gemeinschaft ihr Verhalten ausrichten, um in dieser Gemeinschaft akzeptiert $\mathrm{zu}$ werden. Kultur im anthropologischen Sinne bezieht sich auf erlernte Verhaltensmuster einer Gruppe sowie auf Vorannahmen, Präferenzen und Werte (ebd.).

\section{Literaturverzeichnis}

BUTZKAMM, Wolfgang (2000): Monolingual Principle: In: Byram, Michael (Hrsg.), Routledge Encyclopedia of Language Teaching and Learning, London, New York (2000), S. 415-417: Routledge.

COOK, Guy (1998): Use of Translation in Language Teaching. In Baker, Mona (Hrsg.), Routledge Encyclopedia of Translation Studies, London, New York (1998), S. 117-120: Routledge.

EDMONDSON, Willis; Juliane HOUSE (2006): Einführung in die Sprachlehrforschung, 3. Auflage, Tübingen: Francke.

ENDREß, Gerhard (1982): Einführung in die islamische Geschichte. München: Beck.

FANDRYCH, Christian; Maria THURMAIR (2011): Textsorten im Deutschen. Linguistische Analysen aus sprachdidaktischer Sicht. Tübingen: Stauffenburg.

HALM, Heinz (1994): Die Fatimiden. In: Haarmann, Ulrich (Hrsg.), Geschichte der arabischen Welt, 3. Aufl., München (1994), S. 166-199: Beck.

HARFMANN, Martin. (2010): Explikation in deutsch-arabischer Übersetzung am Beispiel von Imagedarstellungen. Dissertation. http://ediss.sub.uni-hamburg.de/volltexte/2010/4729/ Staats- und Universitätsbibliothek Uni Hamburg.

HOUSE, Juliane. (1997): Translation Quality Assessment. A Model Revisited. Tübingen: Narr.

HOUSE, Juliane. (2001): Übersetzen und Deutschunterricht. Sonderdruck aus: Helbig, Gerhard; Lutz Götze; Gert Henrici, Hans-Jürgen Krumm (Hrsg.), Deutsch als Fremdsprache. Ein internationales Handbuch, Band 19.1., Berlin, New York (2001), S. 258-268: De Gruyter.

HOUSE, Juliane (2002): Möglichkeiten der Übersetzungskritik. In: Best, Joanna; Sylvia Kalina (Hrsg.), Übersetzen und Dolmetschen. Eine Orientierungshilfe. Tübingen, Basel (2002), S. 101-109: Francke.

HOUSE, Juliane (2004): Culture-specific elements in translation. In: Kittel, Harald; Armin Paul Frank, Norbert Greiner, Theo Hermanns, Werner Koller, José Lambert, Fritz Paul (Hrsg.), Übersetzung Translation Traduction. Ein internationals Handbuch zur Übersetzungsforschung. Band 26.1., Berlin, New York (2004), S. 494-504: De Gruyter. 
HOUSE, Juliane (2005): Offene und verdeckte Übersetzung: Zwei Arten, in einer anderen Sprache sdas Gleicher zu sagen. Sonderdruck aus: Zeitschrift für Literaturwissenschaft und Linguistik, Jahrgang 35, Heft 139 (2005), S. 76-101.

HOUSE, Juliane (2010): Übersetzen und Sprachmitteln. In: Krumm, Hans-Jürgen; Christian Fandrych, Britta Hufeisen, Claudia Riemer (Hrsg.), Deutsch als Fremdsprache. Ein internationales Handbuch, Band 1, Berlin, New York (2010), S. 323-331: De Gruyter.

KOLLER, Werner (1995): The Concept of Equivalence and the object of Translation Studies. In: Target 7, 2 (1995), S. 191-222.

KOLLER, Werner (2002): Linguistische und kulturelle Dimension der Übersetzung - in den 70erJahren und heute. In: Thome, Gisela; Claudia Giehl, Heidrun Gerzymisch-Arbogast (Hrsg.), Kultur und Übersetzung, Tübingen (2002), S. 115-130: Gunter Narr.

KOLLER, Werner (2004): Einführung in die Übersetzungswissenschaft. 7. Aufl. Wiebelsheim: Quelle \& Meyer.

KÖNIGS, Frank G. (1994): Psycholinguistische und didaktische Aspekte der Übersetzerausbildung. Neun Thesen zur Reflexion (und zur Provokation). In: Horst Breitung (Hrsg.), Dolmetscherund Übersetzerausbildung. Materialien eines Internationalen Produktionsseminars 17. -21.12. 1993 München, S. 116-136 München: Schubert.

KÖNIGS, Frank G. (2000): Übersetzen im Deutschunterricht? Ja, aber anders! In: Fremdsprache Deutsch 23, S. 6-13.

KÖNIGS, Frank (2010): Übersetzen und Sprachmitteln im Deutsch als Fremdsprache-Unterricht. In Krumm, Hans-Jürgen; Christian Fandrych, Britta Hufeisen, Claudia Riemer (Hrsg.), Deutsch als Fremdsprache. Ein internationales Handbuch, Band 1, Berlin, New York (2010), S. 1040-1047: De Gruyter.

NOTH, Albrecht (1994): Früher Islam. In: Haarman, Ulrich (Hrsg.), Geschichte der arabischen Welt, 3. Aufl., München (1994), S. 11-100: Beck.

SIMONNAES, Ingrid (2002): Interkulturelle Kommunikation und Übersetzungsprobleme. In: Thome, Gisela; Claudia Giehl, Heidrun Gerzymisch-Arbogast (Hrsg.), Kultur und Übersetzung, Tübingen (2002), S. 283-298: Gunter Narr.

STOCK, Kristina. 2005. Arabische Stilistik. Wiesbaden: Reichert Verlag.

WAHRIG. Deutsches Wörterbuch (2006): Wahrig-Burfeind (Hrsg.), 8. Aufl. Gütersloh, München: Bertelsmann.

WEHR, Hans (1985): Arabisches Wörterbuch für die Schriftsprache und Supplement. ArabischDeutsch. Herausg. von Lorenz Kropfitsch, 5. Auflage Wiesbaden: Harrassowitz Verlag.

WEINRICH, Harald. (2007): Textgrammatik der deutschen Sprache. 4. Auflage, Hildesheim, Zürich, New York: Olms. 
Internetquellen

$$
\text { الجامع الأزهر }
$$

http://ar.wikipedia.org/wiki/\%D8\%A7\%D9\%84\%D8\%AC\%D8\%A7\%D9\%85\%D8\%B9 \%D8\%A7\% D9\%84\%D8\%A3\%D8\%B2\%D9\%87\%D8\%B1\#.D8.A7.D9.84.D8.A3.D8.B2.D9.87.D8.B1_.D9.88.D 8.AB.D9.88.D8.B1.D8.A9 .D9.8A.D9.88.D9.84.D9.8A.D9.88 (Zugriff am 13.08. 2011)

$$
\text { جامعة الأزهر }
$$

http://ar.wikipedia.org/wiki/\%D8\%AC\%D8\%A7\%D9\%85\%D8\%B9\%D8\%A9 \%D8\%A7\%D9\%84\% D8\%A3\%D8\%B2\%D9\%87\%D8\%B1 (Zugriff am 13.08. 2011)

$$
\text { قلعة صلاح الدين }
$$

http://ar.wikipedia.org/wiki/\%D9\%82\%D9\%84\%D8\%B9\%D8\%A9 \%D8\%A7\%D9\%84\%D9\%82\% D8\%A7\%D9\%87\%D8\%B1\%D8\%A9 (Zugriff am 14.08. 2011)

Al Azhar Universität

http://de.wikipedia.org/wiki/Al-Azhar-Universit\%C3\%A4t (Zugriff am 15. 09. 2011).

\section{Anhang}

Textteil einer touristischen Broschüre zum Kölner Dom mit historischen Hintergrundinformationen: Deutsches Original und arabische Übersetzung. 


\section{Martin HARFMANN}

Übersetzungsübungen im DaF-Unterricht

Anhang

Textteil einer touristischen Broschüre zum Kölner Dom mit historischen

Hintergrundinformationen: Deutsches Original und arabische Übersetzung.

\section{Geschichte}

Die Anfänge des Kölner Bistums reichen bis ins 4. Jahrhundert zurück Um 313/3/4 wird bereits der erste namentlich bekannte Bischof Maternus erwähnt. Auch haben die Ausgrabungen nach dem 2. Weltkrieg im Dombereich eine Abfolge zahlreicher Um- und Neubauten nachgewiesen. Dem gotischen Neubau unmittelbar vorausgegangen ist eine ca. $95 \mathrm{~m}$ lange, doppelchörige Kirchenanlage, die 870 geweiht wurde. Deren äußere Gestalt ist durch den um 1020 entson 1020 (vistandenen Her.

In dieses Bauwerk überführte 1164 Erzbischof Rainald von Dassel († 1167 ) aus Mailand die Gebeine der Heiligen Drei Könige. Wohl noch unter seinem Nachfolger, Erzbischof Phillipp von Heinsberg († || $9 \mid)$, begann man mit der Herstellung des kostbaren Reliquienschreines, an der der Goldschmied Nikolaus von Verdun allem Anschein nach maßgeblich beteiligt war. Doch erst um 1225 wurde dieses großartige Werk vollendet (vgl. Nr. 19).

Die Reliquien zogen Pilger aus ganz Europa an, die Wallfahrt war eine der größten des Mittelalters. So beschloß das Domkapitel 1248 unter Erzbischof Konrad von Hochstaden $(\dagger|26|)$ einen der Bedeutung der Kathedrat- und Wallfahrtskirche angemessenen Neubau. Hierbei orientierte sich der Bauherr nicht an der romanischen Architektur Deutschlands, sondern an der "modernen“ gotischen Architektur der französischen Kathedralen. Der Gesamtplan úbertraf jedoch in seinen Ausmaßen alle anderen Kirchenbauten seiner Zeit.

Um 1265 waren die Chorkapellen mit dem Chorumgang errichtet. Aus dieser Zeit stammen die ersten Ausstattungsstücke des Chores. Um 1300 war der gesamte Hochchor vollendet, wurde jedoch erst 1322 geweiht. Danach verlangsamte sich das Tempo des Weiterbaus. Erst um 1360 wurde mit dem Bau des südlichen Turmes begonnen, der im mittelalterlicher Zeit nur bis zur Höhe des ersten Obergeschosses fertiggestellt wurde. 1388, dem Jahr der Kölner Universitätsgründung, waren die südllichen Seitenschiffe des Langhauses benutzbar. Ende des 15 . Jahrhunderts wurden noch 7 Felder der nördlichen Seitenschiffe eingewölbt. Im Jahre 1560, als man die Bauarbeiten einstellte, war neben dem fertiggestellten Chorbau zumindest das Endgescho 3 von cehend bentor ant einer Quelle des 15 . Jhn Drenkons bon Dreikonigenschreit 1948 refor Etent we 19. Set 1948 befndet er sich an seenem heutgen Platz. Erst im 19. Jahrhundert wurde das Bauwerk fertiggestellt. 1842 legte Friedrich Winhelm N. von Preußen den Grundstein zum Weiterbau und bereits 1880 setzte man in Anwesenheit Kaiser Wilhelms I. den letzten Stein in die Kreuzblume des Südturms. In dieser Zeit entstanden zahireiche Kunstwerke, die zu den bedeutendsten Schöpfungen der Neugotik gehören.

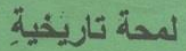

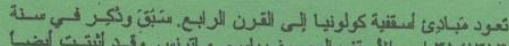

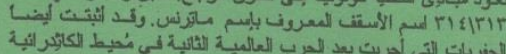

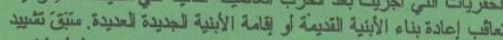

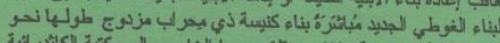

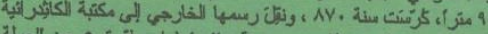

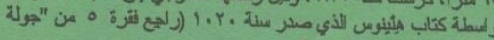

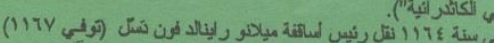

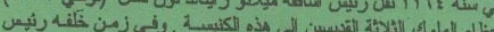

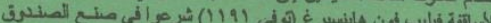

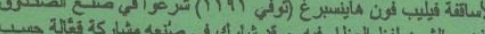

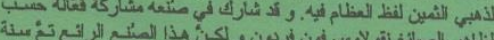

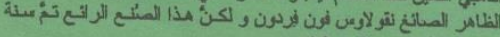

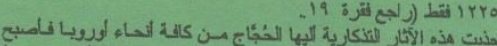

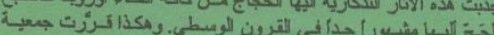

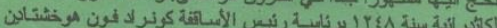

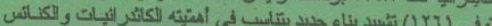

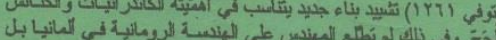

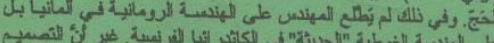

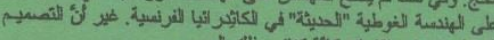

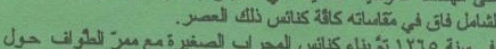

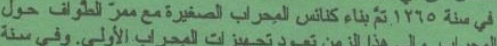

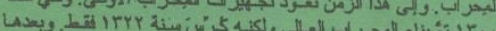

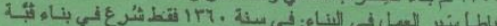

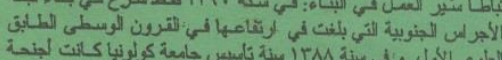

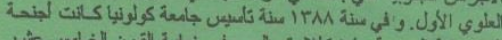

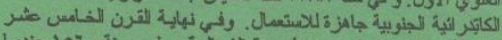

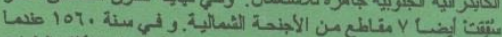

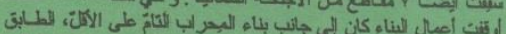

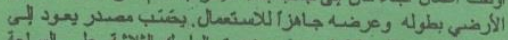

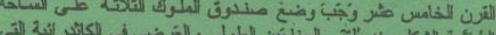

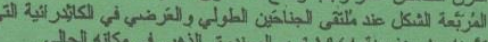

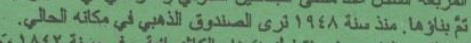

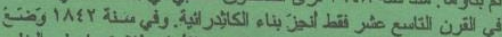

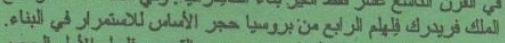

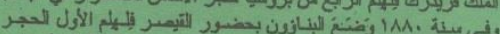

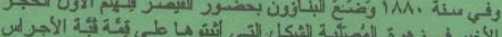

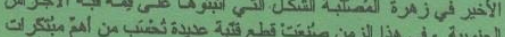

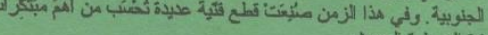

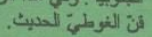

